\title{
MPMI ACKNOWLEDGMENT OF REVIEWERS
}

The success of Molecular Plant-Microbe Interactions depends on the quality of manuscripts submitted by authors and on the care and competence with which they are reviewed. It is the policy of the Editorial Board to solicit reviews of manuscripts from specialists most qualified to review them. In addition to members of the Editorial Board, the individuals listed below provided constructive critical reviews of one or more manuscripts during the past year. Their names are published here in grateful appreciation for their contributions to the journal.

W. Achouak, Commissariat à l'Energie Atomique (CNRS), St. Paul lez Durance, France.

G. K. Agrawal, University Of Missouri-Columbia, Columbia, MO, U.S.A.

C. Allen, University of Wisconsin, Madison, WI, U.S.A.

A. Alspaugh, Duke University Medical Center, Durham, NC, U.S.A.

J.-M. Ané, University of Wisconsin, Madison, WI, U.S.A.

G.-I. Arimura, Max Planck Institute for Chemical Ecology (MPICOE), Jena, Germany

D. Arnold, University of the West of England, Bristol, U.K.

M. Ayliffe, CSIRO Plant Industry, Canberra, Australia

B. A. Bailey, United States Department of Agriculture Agricultural Research Service (USDA ARS), Beltsville, MD, U.S.A.

J. Baker, USDA ARS, Beltsville, MD, U.S.A.

G. Bakkeren, Agriculture \& Agri-Food Canada, Summerland, Canada

I. T. Baldwin, MPICOE, Jena, Germany

I. Barash, Tel-Aviv University, Tel-Aviv, Israel

O. Barazani, Agricultura Research Organization of Israel, Bet Dagan, Israel

M. Barnett, Stanford University, Stanford, CA, U.S.A.

M.-A. Barny, Institut National de la Recherche Agronomique (INRA), Paris, France

C. Baron, McMaster University, Hamilton, Canada

J. Batut, CNRS INRA, Castanet-Tolosan, France

M. Bauscher, ARS, Fort Pierce, FL, U.S.A.

G. Beattie, Iowa State University, Ames, U.S.A.

S. V. Beer, Cornell University, Ithaca, NY, U.S.A.

T. Beliën, K.U.Leuven, Heverlee, Belgium

A. Bendahmane, INRA, Evry, France

C. Bender, Oklahoma State University, Stillwater, U.S.A.

S. Berger, Julius-von-Sachs-Institute of Biosciences, Wuerzburg, Germany

M. K. Bhattacharyya, Iowa State University, Ames, U.S.A.

D. McK Bird, North Carolina State University, Raleigh, U.S.A.

D. M. Bisaro, Ohio State University, Columbus, U.S.A.

J. G. Bishop, Washington State University, Vancouver, U.S.A.

E. Blée, Institut Biologie Moleculaire des Plantes (IBMP), Strasbourg, France

G. Bloemberg, Leiden University, Leiden, The Netherlands

M. Boelker, University of Marburg, Marburg, Germany

M. Bolton, USDA ARS, Saint Paul, MN, U.S.A.

U. Bonas, Martin Luther University, Halle, Germany

L. Borge, Royal Holloway, University of London, Egham, U.K.

M. Brandl, U.S. Department of Agriculture, Albany, CA, U.S.A.

R. W. Briddon, National Institute for Biotechnology and Genetic Engineering, Faisalabad, Pakistan

S. Briggs, University of California, San Diego, CA, U.S.A.

Daniela Buettner, Martin Luther University, Halle, Germany

J. Burgyán, ABC, Godollo, Hungary

T. J. Burr, Cornell University, Geneva, NY, U.S.A.

C. Buschmann, Universityof Hohenheim, Stuttgart, Germany
R. Cameron, McMaster University, Hamilton, Canada

J. Chang, Oregon State University, Corvallis, U.S.A.

T. Charles, University of Waterloo, Waterloo, Canada

A. Chatterjee, University of Missouri, Columbia, MO, U.S.A.

Z. Chen, Purdue University, West Lafayette, IN, U.S.A.

H. Cheng, Lehman College, City University of New York, Bronx, NY, U.S.A.

F. Cillo, Consiglio Nazionale delle Ricerche (CNR), Bari, Italy

L. Ciuffetti, Oregon State University, Corvallis, U.S.A.

S. J. Clough, USDA ARS, Urbana, IL, U.S.A.

A. Collmer, Cornell University, Ithaca, NY, U.S.A.

G. Condemine, CNRS, Villeurbanne, France

U. Conrath, Aachen University, Aachen, Germany

D. Cooksey, University of California, Riverside, CA, U.S.A.

L. Copeland, University of Sydney, Australia

D. Coplin, Ohio State University, Columbus, U.S.A.

K. Craven, Samuel Roberts Noble Foundation, Ardmore, OK, U.S.A.

M. Crespi, CNRS, Gif Sur Yvette, France

J. N. Culver, University of Maryland Biotechnology Institute, College Park, MD, U.S.A.

S. Cunnac, Cornell University, Ithaca, NY, U.S.A.

D. Dalton, Reed College, Portland, OR, U.S.A.

A. M. Dandekar, University of California, Davis, CA, U.S.A.

C. Dardick, Appalachian Fruit Research Station, Kearneysville, WV, U.S.A.

B. Day, Michigan State University, East Lansing, U.S.A.

M. de Bolle, The Centre of Microbial and Plant Genetics, Leuven, Belgium

M. De Vos, Boyce Thompson Institute, Ithaca, NY, U.S.A.

K. Dejonghe, Institute for Agricultural and Fisheries Research, Merelbeke, Belgium

T. P. Denny, University of Georgia, Athens, GA, U.S.A.

C. M. Deom, University of Georgia, Athens, GA, U.S.A.

L. Deslandes, CNRS, Castanet-Tolosan, France

C. Després, Brock University, St. Catharines, Canada

Y. Dessaux, Institut des Sciences du Vegetal, Gif-sur-Yvette, France

M. B. Dickman, Texas A\&M University, College Station, U.S.A.

R. Dickstein, University of North Texas, Denton, U.S.A.

R. G. Dietzgen, Department of Primary Industries \& Fisheries, St. Lucia, Australia

S. P., Dinesh-Kumar, Yale University, New Haven, CT, U.S.A.

M. A. Djordjevic, Australian National University, Canberra, Australia

P. Dodds, CSIRO, Canberra, Australia

X. Dong, Duke University, Durham, NC, U.S.A.

C. Douglas, University of British Columbia, Vancouver, Canada

R. D'Ovidio, University of Tuscia, Viterbo, Italy

I. Dry, CSIRO, Glen Osmond, Australia

L. Du, University of Nebraska, Lincoln, U.S.A.

R. Dudler, University of Zurich, Switzerland

B. Duffy, Agroscope Changins-Wädenswil, Switzerland 
L. Dunkle, USDA ARS, West Lafayette, IN, U.S.A.

S. Duplessis, INRA, Champenoux, France

N. N. Duran-Vila, Instituto Valenciano de Investigationes Agrarias, Moncada, Valencia, Spain

M. Edwards, USDA ARS, Fargo, ND, U.S.A.

C. E. Elliott, School of Botany, Parkville, Australia

P. van Esse, University of Wageningen, The Netherlands

T. Eulgem, University of California, Riverside, U.S.A.

B. Favery, INRA, Sophia-Antipolis, France

G. Felix, Botanisches Institut, Basel, Switzerland

G. Felton, Pennsylvania State University, University Park, U.S.A.

S. Ferrari, Università di Padova, Legnaro, Italy

N. Ferrol, Consejo Superior de Investigaciones Cientificas

(CSIC), Granada, Spain

T. Finan, McMaster University, Hamilton, Canada

V. Flors, University Jaume I, Castellon, Spain

J. Forster, Bundoora, Australia

R. French, USDA ARS, Lincoln, NE, U.S.A.

B. Fristensky, University of Manitoba, Winnipeg, Canada

I. Fudal, INRA, Versailles, France

Y. Gafni, ARO, The Volcani Center, Bet Dagan, Israel

M. Y. Galperin, National Center for Biotechnology Information,

National Institutes of Health, Bethesda, MD, U.S.A.

P. Gamas, INRA-CNRS, Castanet Tolosan, France

S. Gantt, University of Minnesota, St. Paul, U.S.A.

A. Garcia-Brugger, INRA, Dijon, France

S. Gardiner, HortResearch, Palmerston North, New Zealand

W. Gassmann, University of Missouri, Columbia, U.S.A.

C. Gebhardt, MPI for Breeding Research, Cologne, Germany

J. Germida, University Saskatchewan, Saskatoon, Canada

B. Gilbertson, University of California, Davis, U.S.A.

C. Girouss, INRA, Clermont-Ferrand, France

B. Glick, University of Waterloo, Canada

F. Goggin, University of Arkansas, Fayetteville, U.S.A.

S. E. Gold, University of Georgia, Athens, U.S.A.

R. S. Goswami, Simon Fraser University, Burnaby, Canada

M. Göttfert, Technische Universität Dresden, Germany

L. Gout, INRA, Versailles, France

M. Grant, University of Exeter, Devon, U.K.

J. Greenberg, University of Chicago, IL, U.S.A.

P. M. Gresshoff, Centre for Integrative Legume Research,

Brisbane, Australia

F. Grundler, BOKU University, Vienna, Austria

M. L. Guerinot, Dartmouth College, Hanover, NH, U.S.A.

M. Guo, University of Nebraska, Lincoln, U.S.A.

R. S. Gupta, McMaster University, Hamilton, Canada

D. Guttman, University of Toronto, Canada

D. Haas, University of Lausanne, Switzerland

M. Hahn, Kaiserslautern University, Germany

K. Hammond-Kosack, Rothamsted Research, Harpenden, U.K.

A. R. Hardham, Research School of Biological Sciences,

Canberra, Australia

J. Harris, University of Vermont, Burlington, U.S.A.

M. Harrison, Boyce Thompson Institute, Ithaca, NY, U.S.A.

A. Hartmann, GSF-Research Center for Environment and Health GmbH, Neuherberg, Germany

B. Hause, Leibniz Institute of Plant BiochemistryHalle, SachsenAnhalt, Germany

S. Y. He, Michigan State University, East Lansing, U.S.A.

T. Heitz, IBMP, Strasbourg, France

P. Heslop-Harrison, University of Leicester, U.K.

P. Hilson, Department of Plant Systems Biology, Ghent, Belgium

M. Höfte, Ghent University, Belgium

M. Holsters, Ghent University, Belgium

E. B. Holub, University of Warwick, Wellesbourne, U.K.

P. Hooykaas, Institute of Biology Leiden, The Netherlands

G. A. Howe, Michigan State University, East Lansing, U.S.A.
B. Howlett, The University of Melbourne, Parkville, Australia

N. Hugouvieux-Cotte-Pattat, CNRS, Villeurbane, France

S. Hulbert, Washington State University, Pullman, U.S.A.

I. Hwang, Seoul National University, Republic of Korea

M. F. Hynes, University of Calgary, Canada

R. Innes, Indiana University, Bloomington, U.S.A.

C. Ishimaru, University of Minnesota, Saint Paul, U.S.A.

A. Jackson, University of California, Berkeley, U.S.A.

G. Jakab, University of Pecs, Hungary

K. K. Jena, National Institute of Crop Science, Suwon, Kyeonggi Do, Republic of Korea

P. Ji, University of Georgia, Tifton, U.S.A.

D. Joel, Newe-Ya'ar Research Center, Ramat Yishay, Israel

T. Johansson, Lund University, Sweden

D. Jones, Research School of Biological Sciences, Canberra, Australia

L. Jonnsson, Södertörn University College, Huddinge, Sweden

M. H. A. J. Joosten, Wageningen University, The Netherlands

H. Judelson, University of California, Riverside, U.S.A.

I. Jupin, Institut Jacques Monod, Paris, France

P. Kachroo, University of Kentucky, Lexington, U.S.A.

M. Kahn, Washington State University, Pullman, U.S.A.

S. Kamoun, John Innes Centre, Norwich, U.K.

M. Kang, University of Nebraska, Lincoln, U.S.A.

E. Kannenberg, University of Georgia, Athens, U.S.A.

T. Kavanagh, Trinity College Dublin, Ireland

K. Kazan, CSIRO, Brisbane, Australia

B. Keller, University of Zurich, Zurich, Switzerland

H. Keller, INRA, Sophia Antipolis, France

N. Keller, University of Wisconsin, Madison, U.S.A.

H. C. Kistler, University of Minnesota, St. Paul, U.S.A.

D. Klessig, Boyce Thompson Institute, Ithaca, NY, U.S.A.

D. Kliebenstein, University of California, Davis, U.S.A.

J. Kloepper, Auburn University, AL, U.S.A.

R. Koebnik, Martin-Luther-UniversitätInstitut für Genetik, Halle (Saale), Germany

H. Koltai, ARO, Volcani Center, Bet Dagan, Israel

H. Kouchi, National Institute of Agrobiological Sciences, Tsukuba, Japan

T. Kroj, INRA, Castanet-Tolosan, France

C. P. Kubicek, Technische Universität (TU) Wien, ViennaAustria

Y. Kubo, Kyoto Prefectural University, Japan

B. N. Kunkel, Washington University, St. Louis, MO, U.S.A.

J. M. Labavitch, University of California, Davis, U.S.A.

M. Lapidot, Bet Dagan, Israel

C. Lawrence, Virginia Polytechnic Institute and State University, Blacksburg, U.S.A.

O. Le Gall, INRA, Villenave d'Ornon, France

J.-Y. Lee, Delaware Biotechnology Institute, University of Delaware, Newark, U.S.A.

S.-W. Lee, University of California, Davis, U.S.A.

K. Lehto, University of Turku, Finland

S. M. Leisner, University of Toledo, OH, U.S.A.

H. Leung, International Rice Research Institute, Los Banos, Laguna, Philippines

M. G. Lewsey, University of Cambridge, U.K.

X. Li, University of British Columbia, Vancouver, Canada

B. Lievens, Scientia Terrae, Sint-Katelijne-Waver, Belgium

J. Loper, USDA ARS, Corvallis, OR, U.S.A.

I. M. Lopez-Lara, UniversityNacional Autonoma De Mexico (UNAM), Cuernavaca, Mexico

J. Ludwig-Muller, University of Dresden, Germany

M. Lutz, Eidgenössische Technische Hochschule (ETH) Zürich, Switzerland

S. MacFarlane, Scottish Crops Research Institute, Dundee, U.K.

R. L. Mach, TU Wien, Vienna, Austria

D. Mackey, Ohio State University, Columbus, U.S.A. 
K. Mäkinen, University of Helsinki, Finland

J. W. Mansfield, Imperial College at Wye, Ashford, Kent, U.K.

Y. Marco, CNRS INRA, Castanet Tolosan, France

D. Marion, INRA, Nantes, France

G. Martin, Boyce Thompson Institute, Ithaca, NY, U.S.A.

C. Masuta, Hokkaido University, Sapporo, Japan

U. Mathesius, Australian National University, Canberra, Australia

B. Mattei, Università di Roma Sapienza, Italy

A. G. Matthysse, University of North Carolina at Chapel Hill, U.S.A.

F. Mauch, University of Fribourg, Switzerland

B. Mauch-Mani, University of Neuchatel, Switzerland

A. Maule, John Innes Centre, Norwich, U.K.

J. M. McDowell, Virginia Polytechnic Institute, Blacksburg, U.S.A.

H. Meijer, Wageningen University, The Netherlands

K. Mendgen, University of Konstanz, Germany

T. Mengiste, Purdue University, West Lafayette, IN, U.S.A.

J. Michiels, KU Leuven, Heverlee, Belgium

K. Mise, Kyoto University, Japan

T. Mitchell, North Carolina State University, Raleigh, U.S.A.

T. Mitchell-Olds, Duke University, Durham, NC, U.S.A.

M. G. Mitchum, University of Missouri, Columbia, U.S.A.

E. Moriones,CSIC, Algarrobo-Costa, Málaga, Spain

J. Morris, University of Nebraska, Lincoln, U.S.A.

C. Morris, INRA Avignon, Montfavet, France

P. Morris, Bowling Green State University, OH, U.S.A.

L. Mur, University of Wales, Aberystwyth, Wales, U.K.

A. Murphy, University of Cambridge, U.K.

W. Nasser, CNRS-INSA, Villeurbanne, France

U. Nehls, Eberhard-Karls-Universitaet, Tuebingen, Germany

R. Nelson, Samuel Roberts Noble Foundation, Ardmore, OK, U.S.A.

R. L. Nicholson, Purdue University, West Lafayette, IN, U.S.A.

R. E. Niks, Wageningen University, The Netherlands

D. Nino-Liu, Iowa State University, Ames, U.S.A.

J. Nirmala, Washington State University, Pullman, U.S.A.

T. Nürnberger, University Tuebingen, Germany

M. R. O'Brian, State University of New York at Buffalo, U.S.A.

D. Oelofse, Agricultural Research Council, Pretoria, South Africa

V. Oke, University of Pittsburgh, PA, U.S.A.

K. Oparka, University of Edinburgh, U.K.

A. Osbourn, John Innes Centre, Norwich, U.K.

T. Ott, INRA Toulouse, Castanet-Tolosan, France

T. Palva, University of Helsinki, Finland

D. Panaccione, West Virginia University, Morgantown, U.S.A.

R. Panstruga, MPI für Züchtungsforschung, Köln, Germany

I. Parkin, Agric \& Agri-Food Canada, Saskatoon, Canada

K. Pawlowski, Stockholm University, Sweden

K. Pedley, USDA ARS, Ft. Detrick, MD, U.S.A.

N. Peeters, INRA, Castanet-Tolosan, France

A. Penaloza-Vazquez, Oklahoma State University, Stillwater, U.S.A.

M. Perazzolli, Istituto Agrario San Michele, S. Michele all'Adige, Italy

G. S. Pettis, Louisiana State University, Baton Rouge, U.S.A.

L. Pierson III, The University of Arizona, Tucson, U.S.A.

C. M. J. Pieterse, Utrecht University, The Netherlands

M. Piotrowski, Ruhr, Universität Bochum, Germany

G. Podila, University of Alabama, Huntsville, U.S.A.

B. Poinssot, Université de Bourgogne, Dijon, France

M. Pooggin, University of Basel, Switzerland

P. Poole, University of Reading, U.K.

A. L.T. Powell, University of California, Davis, U.S.A.

M. J. Pozo, Estación Experimental del Zaidín, CSIC, Granada, Spain

M. Prins, Wageningen University, The Netherlands
D. Prusky, ARO The Volcani Center, Bet Dagan, Israel

A. Pugin, UMR Université de Bourgogne, Dijon, France

M. Pujol, CIGB, Havana, Cuba

W. Qiu, Missouri State University, Mountain Grove, U.S.A.

R. Raina, Syracuse University, NY, U.S.A.

J. Rathjen, The Sainsbury Laboratory, Norwich, Norfolk, U.K.

M. Rep, University of Amsterdam, The Netherlands

N. Requena, Karlsruhe University, Germany

B. Reuhs, Purdue University, West Lafayette, IN, U.S.A.

F. Revers, INRA, Villenave d'Ornon, France

P. Reymond, University of Lausanne, Switzerland

K. Richards, IBMP, Strasbourg, France

D. Robertson, North Carolina State University, Raleigh, U.S.A.

F. Robertson, University of Cambridge, U.K.

D. Roby, UMR CNRS/INRA Tolosan, Castanet-Tolosan, France

J. A. Rollins, University of Florida, Gainesville, U.S.A.

M. J. Roossinck, The Samuel Roberts Noble Foundation, Ardmore, OK, U.S.A.

S. Rosahl, Leibniz Institute of Plant Biochemistry, Halle (Saale). Germany

J. Rose, Cornell University, Ithaca, NY, U.S.A.

M.-N. Rosso, National Institute for Agricultural Research, Sophia Antipolis, France

T. Rouxel, INRA, Versailles, France

J. Ruiz-Herrera, Centro de Investigación y de Estudios Avanzados del IPN, Irapuato, Mexico

P. Saindrenan, Institut de Biotechnologie des Plantes-CNRS, Orsay, France

G. Salmond, Cambridge University, U.K.

F. Sanchez, Instituto de Biotecnología, UNAM., Cuernavaca, Mexico

N. Sandal, University of Aarhus, Denmark

U. Schaffrath, Institut fuer Biologie III, Aachen, Germany

P. Schenk, University of Queensland, St. Luciam, Australia

N. L. Schlaich, Rheinisch-Westfälische Technische Hochschule Aachen, Germany

H. B. Scholthof, Texas A\&M University, College Station, U.S.A.

A. Schouten, University of Bonn, Germany

H. Schouten, Plant Research International, Wageningen, The Netherlands

D. Shah, Donald Danforth Plant Science Center, St. Louis, MO, U.S.A.

J. Shah, University of Kansas, Manhattan, U.S.A.

R. Shatters, USDA ARS, Ft. Pierce, FL, U.S.A.

J. Shen, University of Nevada, Las Vegas, U.S.A.

W.-B. Shim, Texas A\&M University, College Station, U.S.A.

K. Shirasu, Riken Plant Science Center, Yokohama, Japan

I. Simko, USDA ARS, Salinas, CA, U.S.A.

K. B. Singh, CSIRO, Perth, Australia

P. S. Solomon, Murdoch University, Perth, Australia

I. E Somssich, Max-Planck-Institut fuer Züchtungsforschung, Köln, Germany

R. Sonti, The Centre for Cellular and Molecular Biology, Hyderabad, India

W. Song, University of Florida, Gainesville, U.S.A.

M. J. Soto, Consejo Superior de Investigaciones Científicas, Granada, Spain

J. E. Stajich, University of California, Berkeley, U.S.A.

L. Stavolone, Stituto di Virologia Vegetale, Bari, Italy

D. Stenger, USDA ARS, Parlier, CA, U.S.A.

M. Stevens, Brooms Barn Sugar Beet Research Station, Bury St. Edmunds, Suffolk, U.K.

H. U. Stotz, Oregon State University, Corvallis, U.S.A.

J. Stougaard, University of Aarhus, Denmark

J. Streeter, Ohio State University, Wooster, U.S.A.

A. Sugio, John Innes Centre, Norwich, U.K.

J. T. Sullivan, University of Otago, Dunedin, New Zealand 
G. W. Sundin, Michigan State University, East Lansing, U.S.A.

G. Sunter, University of Texas at San Antonio, San Antonio, U.S.A.

B. Szurek, CNRS, Montpellier, France

K. Suzuki, National Institute of Advanced Industrial Science and Technology, Tsukuba Ibaraki, Japan

L. J. Szabo, USDA ARS, St. Paul, MN, U.S.A.

S. Taira, University of Helsinki, Helsinki, Finland

Y. Takano, Kyoto University, Kyoto, Japan

J.-L. Tang, Guangxi Key Laboratory of Subtropical Bioresources Conservation and Utilization, Nanning, China

D. Tharreau, CIRAD, Montpellier, France

R. Thilmony, USDA ARS, Albany, CA, U.S.A.

H. Thordal-Christensen, Royal Veterinary and Agricultural University, Frederiksberg C, Denmark

L. S. Tisa, University New Hampshire, Durham, U.S.A.

C. Triantaphylides, CEA, St.Paul lez Durance, France

M. Tsagris, University of Crete, Heraklion, Crete, Greece

S. Tsuge, Kyoto Prefectural University, Japan

R. Tuberosa, University of Bologna, Italy

P. Tudzynski, Westfalische Wilhelms University, Münster, Germany

B. Tudzynski, Westfalische Wilhelms University, Münster, Germany

T. Turpen, Biosource Technologies Inc., Vacaville, CA, U.S.A.

T. Uchiumi, Kaogshima University, Japan

M. Udvardi, Samuel Roberts Noble Foundation, Ardmore, OK, U.S.A.

R. Ugalde, UNSAM, San Martí, Buenos Aires, Argentina

S. R. Uppalapati, The Samuel Roberts Noble Foundation, Ardmore, OK, U.S.A.

P. E. Urwin, University of Leeds, U.K.

L. Vaillancourt, University of Kentucky, Lexington, U.S.A.

G. Vale, Consiglio per la Ricerca in Agricoltura (CRA), Fiorenzuola d'Arda, Italy

B. Valent, Kansas State University, Manhattan, U.S.A.

M. Valls, University of Barcelona, Spain

E. Van Damme, Ghent University, Belgium

J. van Kan, Wageningen University, The Netherlands

L. C. Van Loon, Utrecht University, The Netherlands

M.-A. Van Sluys, University of Sao Paulo, Brazil

K. VandenBosch, University of Minnesota, St. Paul, U.S.A.

J. Vanderleyden, KULeuven, Heverlee, Belgium

H. VanEtten, University of Arizona, Tucson, U.S.A.

J. Verchot-Lubicz, Oklahoma State University, Stillwater, U.S.A.
V. Verdier, CNRS, Montpellier, France

D. Vereecke, VIB2-Ghent University, Belgium

Rob Verpoorte, Gorlaeus Labs, Leyden, The Netherlands

B. A. Vinatzer, Virginia Polytechnic Institute, Blacksburg, U.S.A.

P. Vinuesa, Centro de Ciencias Genómicas-UNAM, Cuernavaca, Morelos, Mexico

J. Vivanco, Colorado State University, Fort Collins, U.S.A.

M. Vivier, Stellenbosch University, South Africa

V. Vleeshouwers, Wageningen University, The Netherlands

S. von Bodman, University of Connecticut, Storrs, U.S.A.

J. Vossen, Wageningen University, The Netherlands

F. Waller, Justus-Liebig Universität, Giessen, Germany

J. Walsh, Warwick University, U.K.

G.-L. Wang, The Ohio State University, Columbus, U.S.A.

P. Wang, Research Institute for Children/LSUHSC, New Orleans, LA, U.S.A.

Y. Watanabe, Graduate School of Arts and Sciences, The University of Tokyo, Japan

A. Weissinger, North Carolina State University, Raleigh, U.S.A.

D. Wendehenne, University of Burgundy, Dijon, France

F. F. White, Kansas State University, Manhattan, U.S.A.

C. Williams, USDA ARS Purdue University, West Lafayette, IN, U.S.A.

V. Williamson, University of California, Davis, U.S.A.

M. Wilson, Colorado College, Colorado Springs, U.S.A.

S. Winans, Cornell University, Ithaca, NY, U.S.A.

C. Woloshuk, Purdue University, West Lafayette, IN, U.S.A.

T. Wolpert, Oregon State University, Corvallis, U.S.A.

S.-M. Wong, National University of Singapore, Singapore

J.-r. Xu, Purdue University, West Lafayette, IN, U.S.A.

Y. Yang, Pennsylvania State University, University Park, U.S.A.

B. Yang, Iowa State University, Ames, U.S.A.

C. Yost, University of Regina, Canada

N. Zappel, Max Planck Institute for Plant Breeding Research, Köln, Germany

Y. Zhang, The Samuel Roberts Noble Foundation, Ardmore, OK, U.S.A.

L. Zhang, Institute of Molecular and Cell Biology, Singapore

J. F. Zhang, New Mexico State University, Las Cruces, U.S.A.

T. Z. Zhang, Nanjing Agricultural University, China

J. Zhou, National Institute of Biological Sciences, Beijing, China

H. Ziebell, University of Cambridge, U.K.

V. Ziegler-Graff, IBMP-CNRS, Strasbourg, France

C. Zipfel, The Sainsbury Laboratory, Norwich, U.K. 